\title{
Estratégias de Revascularização em Doentes com Infarto Agudo do Miocárdio em Choque Cardiogênico - Resultados do Registo Português de Síndromes Coronárias Agudas
}

Revascularization Strategies in Patients with Acute Myocardial Infarction and Cardiogenic Shock: Results from the Portuguese Registry on Acute Coronary Syndromes

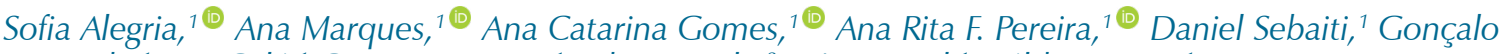 \\ Morgado, ${ }^{1}$ Rita Calé, ${ }^{1}$ Cristina Martins, ${ }^{1}$ Adriana Belo, ${ }^{2}$ Inês Rangel, ${ }^{1}$ Hélder Pereira ${ }^{1}$ \\ Hospital Garcia de Orta EPE, ${ }^{1}$ Almada - Portugal \\ Centro Nacional Coleção de Dados em Cardiologia, ${ }^{2}$ Coimbra - Portugal \\ Em nome dos investigadores do Registo Nacional de Síndromes Coronárias Agudas
}

\section{Resumo}

Fundamento: Em doentes com infarto agudo do miocárdio (IAM), choque cardiogênico (CC) e doença multivaso (DMV) persistem dúvidas sobre a intervenção nas artérias não responsáveis.

Objetivos: 1) caracterizar a amostra de doentes com IAM, CC e DMV incluídos no Registo Nacional Português de Síndromes Coronárias Agudas (RNSCA); 2) comparar os eventos associados a diferentes estratégias de revascularização; e 3) identificar preditores de mortalidade intra-hospitalar nesta amostra.

Métodos: Estudo observacional retrospetivo de doentes com IAM, CC e DMV incluídos no RNSCA entre 2010 e 2018. Compararam-se duas estratégias de revascularização: completa durante o procedimento índice (grupo 1); e completa diferida ou incompleta durante o internamento (grupo 2-3). O endpoint primário foi a ocorrência de reinfarto ou morte intra-hospitalar. A significância estatística foi definida por um valor $p<0,05$.

Resultados: Identificaram-se 127 doentes com IAM, CC e DMV (18,1\% no grupo 1 e 81,9\% no grupo 2-3), com idade média de $70 \pm 12$ anos e 92,9\% com IAM com supradesnivelamento do segmento ST. O endpoint primário ocorreu em $47,8 \%$ dos doentes do grupo 1 e em 37,5\% do grupo 2-3 ( $p=0,359$ ). As taxas de mortalidade intra-hospitalar, reinfarto, acidente vascular cerebral e hemorragia major foram também semelhantes nos dois grupos. Os preditores de mortalidade intra-hospitalar nesta amostra foram a presença na admissão de disfunção ventricular esquerda (OR 16,8), bloqueio completo de ramo direito (OR 7,6) e anemia (OR 5,2), ( $p \leq 0,02)$.

Conclusões: Entre os doentes com IAM, CC e DMV, incluídos no RNSCA, não se verificou diferença significativa entre revascularização completa no evento índex e completa diferida ou incompleta durante o internamento, relativamente à ocorrência de morte intra-hospitalar ou reinfarto. (Arq Bras Cardiol. 2021; 116(5):867-876)

Palavras-chave: Infarto do Miocárdio; Choque Cardiogênico; Revascularização Miocárdica; Síndrome Coronária Aguda; Disfunção Ventricular Esquerda; Mortalidade; Biomarcadores; Hemorragia Intracraniana.

\footnotetext{
Abstract

Background: In patients with acute myocardial infarction (MI), cardiogenic shock (CS), and multivessel disease (MVD) questions remain unanswered when it comes to intervention on non-culprit arteries.

Objective: This article aims to 1) characterize patients with MI, CS and MVD included in the Portuguese Registry on Acute Coronary Syndromes (ProACS); 2) compare different revascularization strategies in the sample; 3) identify predictors of in-hospital mortality among these patients.

Methods: Observational retrospective study of patients with MI, CS and MVD included in the ProACS between 2010 and 2018. Two revascularization strategies were compared: complete during the index procedure (group 1); and complete or incomplete during the index hospitalization (groups 2-3). The primary endpoint was a composite of in-hospital death or MI. Statistical significance was defined by a p-value $<0.05$.
}

Correspondência: Sofia Alegria •

Avenida Torrado da Silva 2805-267, Almada - Portugal

Email: asofia.alegria@gmail.com

Artigo recebido em 25/10/2019, revisado em 11/03/2020, aceito em 15/04/2020

DOI: https://doi.org/10.36660/abc.20190739 
Results: We identified 127 patients with MI, CS, and MVD (18.1\% in group 1, and 81.9\% in groups 2-3), with a mean age of 7012 years, and 92.9\% of the sample being diagnosed with ST-segment elevation MI (STEMI). The primary endpoint occurred in $47.8 \%$ of the patients in group 1 and $37.5 \%$ in group 2-3 ( $p=0.359)$. The rates of in-hospital death, recurrent MI, stroke, and major bleeding were also similar. The predictors of in-hospital death in this sample were the presence of left ventricle systolic dysfunction on admission (OR 16.8), right bundle branch block (OR 7.6), and anemia (OR 5.2) ( $p \leq 0.02$ for both).

Conclusions: Among patients with MI, CS, and MVD included in the ProACS, there was no significant difference between Complete and incomplete revascularization during the index hospitalization regarding the occurrence of in-hospital death or MI. (Arq Bras Cardiol. 2021; 116(5):867-876)

Keywords: Myocardial Infarction; Shock, Cardiogenic; Myocardial Revascularization; Acute Coronary Syndrome; Ventricular Dysfunction ,Left; Mortality; Biomarkers; Intracranial Hemorrhage.

Full texts in English - http://www.arquivosonline.com.br

\section{Introdução}

Nos doentes que se apresentam com infarto agudo do miocárdio (IAM) em choque cardiogênico (CC), a revascularização da artéria responsável associa-se a uma melhoria do prognóstico. ${ }^{1}$ No entanto uma proporção significativa destes doentes apresenta doença multivaso $(\mathrm{DMV})^{2}$ o que levanta a questão sobre a indicação e timing para revascularização das artérias não responsáveis.

As recomendações da Sociedade Europeia de Cardiologia (ESC) sobre abordagem do IAM com supradesnivelamento do segmento ST (IAMCSST) publicadas em 2017 defendiam a revascularização imediata das artérias não responsáveis nos doentes em CC (recomendação classe Ila, nível de evidência C). ${ }^{3}$

No entanto, os resultados do ensaio clínico Culprit-Shock, publicado no mesmo ano, desafiaram esta recomendação. ${ }^{4}$ Este estudo incluiu 706 doentes com IAM, CC e DMV, aleatorizados para duas estratégias de revascularização percutânea: angioplastia da artéria responsável, com a opção de revascularização diferida dos restantes vasos durante o internamento, ou angioplastia multivaso imediata. Os resultados demonstraram que o endpoint combinado de morte ou lesão renal grave com necessidade de técnica de substituição renal foi significativamente mais baixo nos doentes submetidos inicialmente apenas a angioplastia da artéria responsável. ${ }^{4}$

Estes dados foram fundamentais na alteração das recomendações mais recentes. Assim, as recomendações da ESC sobre revascularização miocárdica publicadas em 2018 atribuem uma recomendação classe III a esta estratégia. ${ }^{5}$

Desta forma, os objetivos deste trabalho foram: 1) caracterizar a amostra de doentes com IAM, CC e DMV incluídos no Registo Nacional Português de Síndromes Coronárias Agudas (RNSCA); 2) comparar os eventos associados a diferentes estratégias de revascularização; e 3) identificar preditores de mortalidade intra-hospitalar nesta amostra.

\section{Métodos}

Estudo observacional com análise retrospetiva de doentes admitidos com IAM, apresentação em CC (classe Killip-Kimball IV) e DMV, incluídos no RNSCA entre Outubro de 2010 e Janeiro de 2018.
Foram comparadas três estratégias de revascularização: revascularização completa durante o evento índice - grupo 1; revascularização completa diferida durante o internamento - grupo 2; e revascularização incompleta durante o internamento - grupo 3.

Para a definição de lesão coronária significativa foram utilizados critérios angiográficos, considerando-se como significativa uma lesão com estenose igual ou superior a 50\%. Considerou-se revascularização completa quando todas as lesões coronárias significativas foram revascularizadas.

\section{Definição de IAM}

O IAM foi definido de acordo com o documento de definição de variáveis do Registo Nacional de Síndromes Coronárias Agudas.6

O IAM com Supradesnivelamento do segmento ST foi definido pela presença de supradesnivelamento persistente (> 30 minutos) do segmento ST $>1 \mathrm{~mm}(0,1 \mathrm{mV})$ em duas ou mais derivações contíguas ou bloqueio completo de ramo esquerdo (BCRE) de novo, em contexto clínico sugestivo de isquemia miocárdica.

O IAM sem Supradesnivelamento do segmento ST foi definido pela ausência de supradesnivelamento persistente (< 30 minutos) do segmento ST associada a elevação de biomarcadores de necrose miocárdica (troponina ou CK- mb) em contexto clínico sugestivo de isquemia miocárdica.

\section{Definição dos endpoints}

Definiu-se como endpoint primário combinado a ocorrência de reinfarto ou morte intra-hospitalar. Os endpoints foram definidos de acordo com o documento de definição de variáveis do Registo Nacional de Síndromes Coronárias Agudas. ${ }^{6}$ Reinfarto foi definido pela recorrência de dor torácica sugestiva de isquemia, após resolução do episódio de dor da admissão, com duração superior a 20 minutos, acompanhada de alterações eletrocardiográficas e de nova elevação dos biomarcadores de necrose miocárdica em relação ao valor prévio (elevação de CK-MB duas vezes o valor de referência ou > 50\% do valor prévio; ou elevação > 20\% do valor da Troponina I/T em relação ao valor prévio).

Acidente vascular cerebral (AVC) isquémico foi definido pela instalação de novo de défices neurológicos focais sem evidência de hemorragia na tomografia computorizada (TC) cerebral durante o internamento hospitalar, e o AVC 
hemorrágico pela instalação durante o internamento hospitalar de défices neurológicos focais de novo com evidência de hemorragia na TC cerebral. A definição de complicação mecânica de IAM incluiu ruptura de parede livre, ruptura do septo interventricular e insuficiência mitral aguda grave, por envolvimento dos músculos papilares.

Hemorragia major durante o internamento hospitalar foi definida de acordo com a classificação GUSTO (hemorragia intracraniana ou hemorragia com compromisso hemodinâmico requerendo intervenção). ${ }^{7}$

\section{Análise Estatística}

A caracterização das variáveis contínuas foi feita recorrendo a média amostral e desvio-padrão, ou mediana e intervalo interquartil, conforme a presença ou não de normalidade na distribuição dos dados. A comparação das médias foi realizada recorrendo ao teste t-Student não pareado ou ao teste não paramétrico Mann-Whitney. A normalidade foi testada com o teste de Kolmogorov-Smirnov. As variáveis categóricas foram caracterizadas por meio de percentagens e as associações entre grupos foram analisadas pelo teste de $X^{2}$ ou pelo teste de Fisher, conforme apropriado. Foi ajustado um modelo de regressão logística multivariada para identificação dos preditores independentes de mortalidade intra-hospitalar, com ajuste para variáveis demográficas, diagnóstico, localização do IAMCSST, fatores de risco, antecedentes, frequência cardíaca, pressão arterial, ritmo, morfologia dos complexos QRS, artérias coronárias com lesão, função ventricular esquerda, dados laboratoriais e medicação prévia e intra-hospitalar.

Para a análise estatística foi utilizado o software SPSS 19.0.0.2. Um valor de $p<0,05$ foi considerado estatisticamente significativo.

\section{Resultados}

\section{Caracterização da Amostra}

Entre os 17.834 doentes incluídos no RNSCA entre Outubro de 2010 e Janeiro de 2018, identificaram-se 222 doentes com IAM e CC na admissão, submetidos a angioplastia $(1,2 \%)$ (Figura 1). Destes, 57,2\% $(n=127)$ apresentavam DMV tendo sido incluídos na análise $(18,1 \%$ no grupo $1, \mathrm{n}=23$; $3,1 \%$ no grupo $2, n=4 ; 78,7 \%$ no grupo $3, n=100$ ).

A caracterização da amostra encontra-se detalhada nas tabelas 1-4. Os doentes apresentavam uma idade média de 7012 anos e predomínio do sexo masculino $(68,5 \%, \mathrm{n}=$ 87). Cerca de três quartos (72,5\%) apresentavam história de hipertensão arterial, 33,1\% de diabetes mellitus, 57,5\% de dislipidemia, 23,0\% de tabagismo, $14,5 \%$ de IAM e $8,2 \%$ de doença renal crónica; 4,2\% apresentavam história familiar de doença coronária prematura.

Cerca de um terço dos doentes $(36,3 \%, \mathrm{n}=45)$ foram admitidos em centros sem Cardiologia de Intervenção e $28,6 \%(n=30)$ recorreram ao serviço de urgência por meios próprios. A maioria apresentou diagnóstico de IAM com supradesnivelamento do segmento ST (IAMCSST) (92,9\%), 6,3\% IAM sem supradesnivelamento do segmento ST (IAMSSST) e 0,8\% IAM de origem indeterminada. A artéria responsável foi o tronco comum em $17,2 \%$, a descendente anterior em $25,9 \%$, a circunflexa em $10,3 \%$ e a coronária direita em $35,3 \%$ dos casos. Utilizou-se balão intra-aórtico em $18,9 \%$ dos doentes ( $n=24$ ) e em nenhum doente foi implantado um dispositivo de assistência ventricular, enquanto $37,0 \%$ necessitaram de ventilação mecânica invasiva $(n=47)$. O endpoint primário ocorreu em 39,4\% dos doentes $(n=50)$ e a taxa de mortalidade intra-hospitalar foi de $37,8 \%(n=48)$.

\section{Comparação entre Estratégias de Revascularização}

Considerando o pequeno número de doentes no grupo 2, a comparação das estratégias de revascularização foi realizada entre os grupos 1 e o grupo 2-3 (revascularização completa no procedimento índice vs. completa diferida ou incompleta durante o internamento), correspondendo a $18,1 \%$ dos doentes no grupo $1(n=23)$ e $81,9 \%$ no grupo $2-3(n=104)$.

Na comparação entre grupos constatou-se que os doentes do grupo 1 eram mais novos $(63 \pm 10$ vs. $72 \pm 12$ anos, $\mathrm{p}<0,001)$ e apresentavam maior prevalência de hábitos tabágicos (45,5 vs. 18,0\%, p=0,006); na admissão tinham maior prevalência de ritmo sinusal $(95,7$ vs. $76,0 \%, p=0,043)$, valores de hemoglobina $(\mathrm{Hg})$ mais altos $(14,28 \mathrm{gr} / \mathrm{dl}$ vs. $13,1 \pm 1,9 \mathrm{gr} / \mathrm{dl}, \mathrm{p}=0,033$ ) e apresentavam um valor de péptido natriurético cerebral (brain natriuretic peptide - BNP) mais baixo (mediana 88; intervalo interquartis (IIQ) 34-535 vs. 455,5; IIQ 176,5-1234,5 pg/ml), p=0,040 (Tabelas 1 e 2). O infarto anterior foi mais prevalentes no grupo 1 $(72,7 \%$ vs. $45,8 \%, p=0,023)$ e o infarto inferior no grupo $3(13,6 \%$ vs. $52,1 \%, p=0,001)$ (Tabela 2). Relativamente à anatomia coronária constatou-se ainda que os doentes do grupo 1 apresentavam todos doença de dois vasos, pelo que a prevalência de doença de três vasos foi superior no grupo $2-3(0,0 \%$ vs. $48,9 \%, p<0,001)$. Por outro lado, o tronco comum foi mais frequente como artéria responsável no grupo 1 (40,0 vs. 12,5\%; $p=0,007$ ), enquanto a coronária direita foi mais frequente no grupo 3 (5,0 vs. 41,7\%; $p=0,002$ ) (Tabela 3). De referir ainda que não houve diferenças entre o valor de creatinina $(\mathrm{Cr})$ máxima durante o internamento nos dois grupos (Tabela 2).

O endpoint primário ocorreu em 47,8\% ( $n=11)$ dos doentes do grupo 1 e em $37.5 \%(n=39)$ do grupo $2-3$ $(p=0,359)$. As taxas de mortalidade intra-hospitalar, reinfarto, AVC e hemorragia major (definida de acordo com os critérios GUSTO) também foram semelhantes entre os dois grupos, embora tenha havido maior incidência de bloqueio auriculoventricular de $2^{\mathrm{O}}$ grau Mobitz II ou de 3 o grau no grupo 2-3 (8,7 vs. 31,7\%; $p=0,025$ ) (Tabela 4 e Figura 2).

\section{Preditores de Mortalidade Intra-Hospitalar}

$\mathrm{Na}$ análise de regressão logística multivariada os preditores independentes de mortalidade intra-hospitalar foram a presença de disfunção ventricular esquerda (fração de ejeção inferior a 40\%) (OR 16,79; IC 95\% 5,03-56,02; p = 0,001), a presença de bloqueio completo de ramo direito (BCRD) (OR 7,60; IC 95\% 2,22-25,97; p = 0,001) e hemoglobina inferior a $12 \mathrm{~g} / \mathrm{dl}$ na admissão (OR 5,18; IC 95\% 1,82-14,76; $\mathrm{p}=0,002$ ) (Tabela 5). 


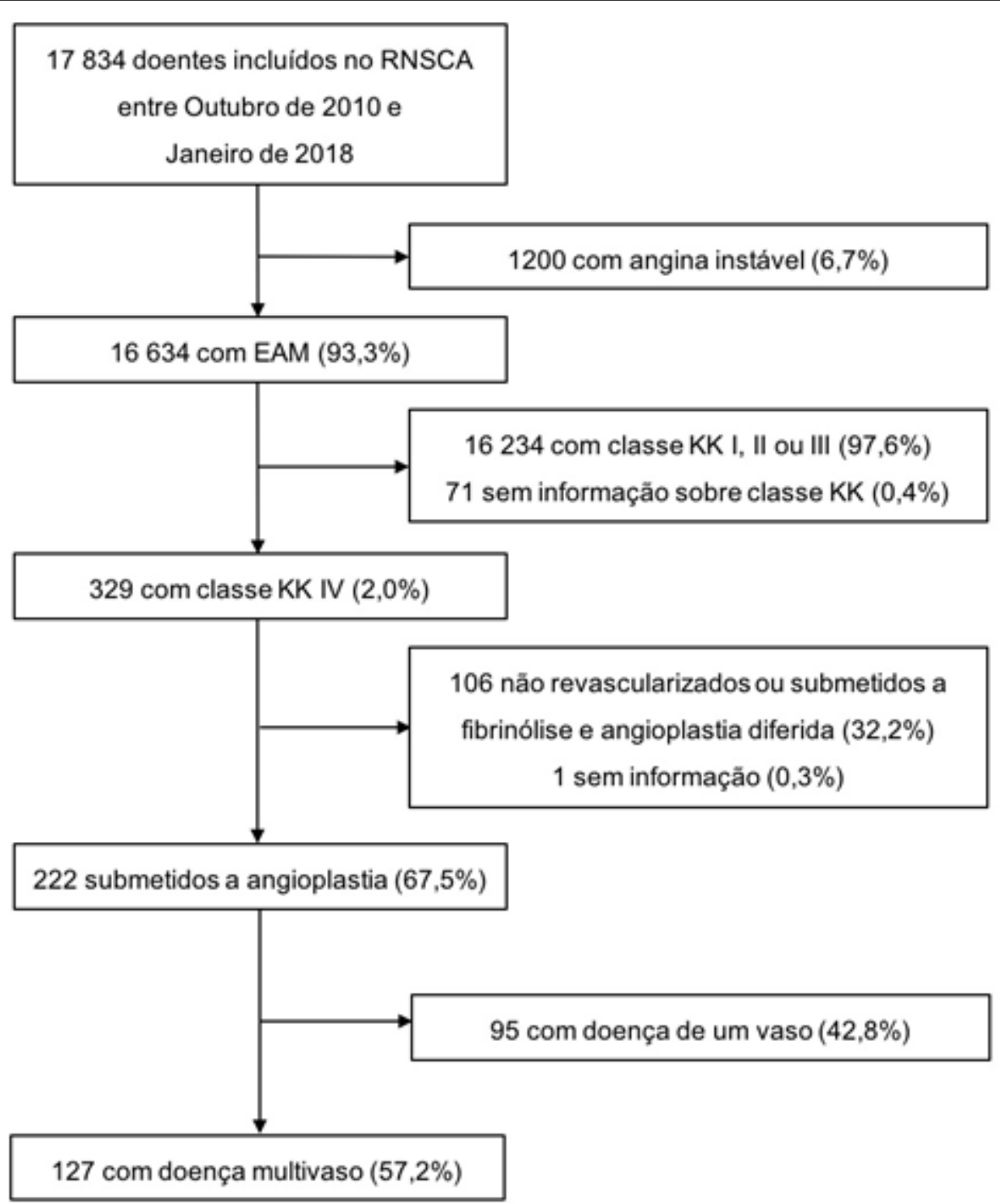

Figura 1 - Fluxograma de inclusão de doentes na análise. IAM: infarto agudo do miocárdio; KK: Killip-Kimball; RNSCA: Registo Nacional de Síndromes Coronárias Agudas.

\section{Discussão}

A DMV é frequente nos doentes com IAM e está relacionada com pior prognóstico, incluindo aumento de mortalidade. ${ }^{8}$ Este estudo, que incluiu uma amostra de doentes com IAM, CC na admissão e DMV, incluídos no RNSCA, mostra que, ao contrário do que defendiam as recomendações anteriores, a prática mais frequente era a realização de revascularização apenas da artéria responsável no procedimento índice.

Por outro lado, os resultados sugerem que a realização de revascularização completa no procedimento índice em comparação com revascularização diferida no mesmo internamento ou incompleta durante o internamento, não apresentou diferenças significativas relativamente ao endpoint combinado de reinfarto ou morte intra-hospitalar, pelo que esta estratégia parece ser segura.
Na comparação entre grupos constatou-se que os doentes submetidos a revascularização completa eram mais novos, na admissão estavam mais frequentemente em ritmo sinusal, apresentavam valores de $\mathrm{Hg}$ mais altos e um valor de BNP mais baixo, ou seja, eram doentes mais jovens, menos frágeis e provavelmente apresentavam quadros menos graves e, consequentemente, com menor risco para revascularização completa, nomeadamente em relação à ocorrência de nefropatia de contraste.

Nos doentes com IAM e DMV a angioplastia da lesão responsável é de facto o standard of care, mas a abordagem das restantes lesões tem sido um tema bastante controverso. Os resultados dos últimos ensaios clínicos aleatorizados, incluindo o PRAMI (Preventative angioplasty in acute myocardial 


\begin{tabular}{|c|c|c|c|c|}
\hline & $\begin{array}{l}\text { Amostra } \\
(n=127)\end{array}$ & $\begin{array}{l}\text { Grupo } 1 \\
(n=23)\end{array}$ & $\begin{array}{c}\text { Grupo 2-3 } \\
(n=104)\end{array}$ & Valor $p^{*}$ \\
\hline Idade (anos) - média \pm DP & $70 \pm 12$ & $63 \pm 10$ & $72 \pm 12$ & $<0,001$ \\
\hline Sexo masculino (\%) & 68,5 & 78,3 & 66,3 & 0,266 \\
\hline IMC (Kg/m2) & $26,9 \pm 4,2$ & $28,2 \pm 4,8$ & $26,5 \pm 4,0$ & 0,081 \\
\hline Fumador (\%) & 23,0 & 45,5 & 18,0 & 0,006 \\
\hline HTA (\%) & 72,5 & 66,7 & 73,7 & 0,510 \\
\hline Diabetes mellitus (\%) & 33,1 & 27,3 & 34,3 & 0,504 \\
\hline Dislipidemia (\%) & 57,5 & 66,7 & 55,4 & 0,347 \\
\hline $\begin{array}{l}\text { História familiar de doença } \\
\text { coronária prematura (\%) }\end{array}$ & 4,2 & 10,5 & 2,6 & 0,174 \\
\hline IAM prévio (\%) & 14,5 & 13,6 & 14,7 & 1,000 \\
\hline ICP prévia (\%) & 10,3 & 13,0 & 9,7 & 0,704 \\
\hline $\begin{array}{l}\text { Cirurgia de revascularização } \\
\text { miocárdica prévia (\%) }\end{array}$ & 1,6 & 0,0 & 1,9 & 1,000 \\
\hline AIT/AVC prévio (\%) & 15,1 & 4,3 & 17,5 & 0,194 \\
\hline Doença vascular periférica (\%) & 5,7 & 4,3 & 6,0 & 1,000 \\
\hline DRC (\%) & 8,2 & 13,0 & 7,1 & 0,397 \\
\hline
\end{tabular}

* Comparação entre revascularização completa e revascularização incompleta. AlT: acidente isquémico transitório; AVC: acidente vascular cerebral; DP: desvio padrão; DRC: doença renal crónica; IAM: infarto agudo do miocárdio; HTA: hipertensão arterial; ICP: intervenção coronária percutânea; IMC: índice de massa corporal.

infarction), ${ }^{9}$ o CVLPRIT (Complete versus lesion-only primary PCI trial), ${ }^{10}$ o DANAMI-3 PRIMULTI (The third danish study of optimal acute treatment of patients with ST-segment elevation myocardial infarction: primary $\mathrm{PCl}$ in patients with ST-elevation myocardial infarction and multivessel disease $)^{11}$ e o COMPARE-ACUTE (Comparison between FFR guided revascularization versus conventional strategy in acute STEMI patients with MVD) trial, ${ }^{12}$ sugerem que a revascularização completa destes doentes poderá ser benéfica, contribuindo, por exemplo, para a recuperação da função ventricular e do estado hemodinâmico. ${ }^{3} \mathrm{Na}$ realidade, o argumento para a revascularização completa baseia-se no potencial de melhorar a perfusão e função miocárdica global, embora a sua realização no evento índice coloque outros problemas adicionais, nomeadamente a indução de mais isquemia, sobrecarga de volume e agravamento da função renal associada ao aumento da dose de contraste utilizada. ${ }^{8}$ Salienta-se que em todos os estudos ocorreu redução do endpoint primário no grupo de revascularização completa, à custa sobretudo da redução da necessidade de revascularização adicional e da ocorrência de IAM não fatal, mas sem redução significativa da mortalidade.

Relativamente ao melhor timing de revascularização das artérias não responsáveis (imediata vs. diferida) não existe ainda evidência suficiente, sendo que nos ensaios foram utilizadas diferentes estratégias: revascularização das artérias não responsáveis no procedimento índice (PRAMI and Compare-Acute), ${ }^{9,12}$ revascularização diferida durante o internamento (DANAMI-3-PRIMULTI), ${ }^{11}$ ou em qualquer altura antes da alta (imediata ou diferida) (CVLPRIT). ${ }^{10}$
Neste contexto, em 2017, as recomendações de IAMCSST do ESC atualizaram as indicações sobre a estratégia de revascularização nos doentes com DMV, atribuindo uma recomendação classe $\mathrm{II}$, nível de evidência $\mathrm{A}$, à revascularização completa por rotina antes da alta hospitalar. ${ }^{3}$

No entanto, os doentes em CC não foram incluídos nestes últimos ensaios. Já o ensaio CULPRIT-SHOCK ${ }^{4}$ (Culprit lesion only $\mathrm{PCl}$ versus multivessel $\mathrm{PCl}$ in cardiogenic shock) demonstrou que, em doentes com IAM e CC, o tratamento por rotina das lesões não responsáveis durante a angioplastia primária se associou a um aumento do endpoint combinado de mortalidade e lesão renal aguda grave com necessidade de técnica de substituição renal. Com base nestes resultados, as últimas recomendações do ESC sobre revascularização miocárdica, publicadas em 2018, consideram que a revascularização das artérias não responsáveis durante a angioplastia primária nestes doentes não deve ser realizada, sendo uma recomendação de classe III. ${ }^{5}$

Tendo em conta a evidência mais recente é também da maior relevância avaliar os dados da vida real. No presente estudo, os doentes submetidos a revascularização completa no evento índex apresentaram maior taxa de mortalidade intra-hospitalar e do endpoint combinado de morte intrahospitalar ou reinfarto, embora esta diferença não tenha atingido significado estatístico (43,5 vs. 36,5\%, $p=0,535$; 47,8 vs. $37,5 \%, p=0,359$, respetivamente). A ocorrência de reinfarto, AVC ou hemorragia major foram semelhantes entre os dois grupos. Comparativamente, o ensaio Culprit-Shock 


\begin{tabular}{|c|c|c|c|c|}
\hline & $\begin{array}{l}\text { Amostra } \\
(n=127)\end{array}$ & $\begin{array}{l}\text { Grupo } 1 \\
(\mathrm{n}=23)\end{array}$ & $\begin{array}{c}\text { Grupo 2-3 } \\
(n=104)\end{array}$ & Valor $\mathrm{p}^{*}$ \\
\hline Transporte pela VMER (\%) & 32,4 & 52,9 & 28,4 & 0,048 \\
\hline Transporte por ambulância sem médico (\%) & 25,7 & 11,8 & 28,4 & 0,227 \\
\hline Transporte por meios próprios (\%) & 28,6 & 17,6 & 30,7 & 0,384 \\
\hline Transporte por outros meios (\%) & 13,3 & 17,7 & 12,5 & 0,462 \\
\hline $\begin{array}{l}\text { Admissão em hospital sem laboratório de } \\
\text { Hemodinâmica (\%) }\end{array}$ & 36,3 & 30,4 & 37,6 & 0,518 \\
\hline $\begin{array}{l}\text { Tempo sintomas-admissão (minutos) - } \\
\text { mediana (IIQ) }\end{array}$ & $152(82-270)$ & $130(90-223)$ & $154(79-271)$ & 0,387 \\
\hline IAMCSST (\%) & 92,9 & 95,7 & 92,3 & 1,000 \\
\hline IAMSSST (\%) & 6,3 & 4,3 & 6,7 & 1,000 \\
\hline IAM indeterminado (\%) & 0,8 & 0,0 & 1,0 & 1,000 \\
\hline IAM de localização anterior (\%) & 50,8 & 72,7 & 45,8 & 0,023 \\
\hline IAM de localização inferior (\%) & 44,9 & 13,6 & 52,1 & 0,001 \\
\hline FC (bpm) - média $\pm \mathrm{DP}$ & $82 \pm 33$ & $93 \pm 36$ & $80 \pm 32$ & 0,162 \\
\hline PAS (mmHg) - média \pm DP & $93 \pm 27$ & $90 \pm 27$ & $94 \pm 27$ & 0,446 \\
\hline Fibrilhação auricular (\%) & 10,2 & 4,3 & 11,5 & 0,460 \\
\hline Creatinina (mg/dl) - mediana (IIQ) & $1,2(0,9-1,7)$ & $1,5(0,8-2,0)$ & $1,2(1,0-1,7)$ & 0,835 \\
\hline Creatinina máxima $(\mathrm{mg} / \mathrm{dl})$ - mediana $(I I Q)$ & $1,6(1,1-2,6)$ & $1,6(1,2-2,8)$ & $1,6(1,1-2,6)$ & 0,731 \\
\hline Hemoglobina $(\mathrm{g} / \mathrm{dl})$ - média $\pm \mathrm{DP}$ & $13,3 \pm 1,9$ & $14,2 \pm 1,8$ & $13,1 \pm 1,9$ & 0,033 \\
\hline BNP $(\mathrm{pg} / \mathrm{mL})$ - mediana (IIQ) & $388(100-779)$ & $88(34-535)$ & $456(177-1235)$ & 0,040 \\
\hline Função VE < $40 \%$ (\%) & 61,0 & 77,8 & 57,3 & 0,107 \\
\hline
\end{tabular}

demonstrou superioridade com a revascularização apenas da artéria responsável (com a possibilidade de revascularização completa diferida) com redução do endpoint combinado de mortalidade a 30 dias ou lesão renal aguda grave com necessidade de técnica de substituição renal (43,3 vs. 51,6\%; HR 0.84, IC 95\% 0,72-0,98; $p=0,03$ ) e da mortalidade a 30 dias. ${ }^{4}$ No RNSCA não existe informação sobre necessidade de técnica de substituição renal pelo que não foi possível analisar este evento, embora se tenha constatado que o valor de creatinina máxima durante o internamento não foi diferente na comparação dos grupos em estudo.

Existem ainda outros aspetos que carecem de melhor esclarecimento, nomeadamente no que diz respeito à identificação das lesões não responsáveis que beneficiam de revascularização (angiografia, avaliação funcional ou imagiologia intracoronária) e ao melhor timing da realização do procedimento diferido. Na realidade, nos principais ensaios aleatorizados a decisão de angioplastia dos vasos não responsáveis foi guiada de diferentes formas, nomeadamente por angiografia com decisão de intervir em lesões com estenose superior a 50\% (PRAMI). ${ }^{9}$ ou superior a $70 \%$ (CVLPRIT), ${ }^{10}$ ou ainda por avaliação funcional guiada por fractional flow reserve (FFR) (DANAMI-3-PRIMULTI e Compare-Acute). ${ }^{11,12}$

Neste trabalho, o facto da amostra de doentes que realizou revascularização completa diferida durante o internamento ser pequena condicionou a comparação das estratégias, não sendo possível avaliar a presença de diferenças entre a revascularização completa no evento índice versus revascularização completa diferida. No ensaio Culprit-Shock efetivamente houve possibilidade de revascularização completa durante a angioplastia primária versus revascularização apenas da artéria responsável com a possibilidade de revascularização diferida das restantes artérias. Salienta-se, no entanto, que neste último grupo apenas foi realizada revascularização diferida durante o internamento em aproximadamente $18 \%$ dos doentes. ${ }^{4}$ De forma semelhante, diversas meta-análises incluindo estudos aleatorizados e não aleatorizados com doentes com IAMCSST com ou sem CC também demonstraram mortalidade semelhante ou superior com revascularização completa num 


\section{Tabela 3 - Terapêutica / procedimentos intra-hospitalares}

\begin{tabular}{|c|c|c|c|c|}
\hline & $\begin{array}{l}\text { Amostra } \\
(n=127)\end{array}$ & $\begin{array}{c}\text { Grupo } 1 \\
(n=23)\end{array}$ & $\begin{array}{c}\text { Grupo } 2-3 \\
(n=104)\end{array}$ & valor $p^{*}$ \\
\hline Aspirina (\%) & 96,1 & 91,3 & 97,1 & 0,222 \\
\hline Clopidogrel (\%) & 84,1 & 73,9 & 86,4 & 0,202 \\
\hline Ticagrelor (\%) & 16,8 & 23,5 & 15,4 & 0,476 \\
\hline Inibidores GP IIb-IIIa & 37,6 & 52,2 & 34,3 & 0,110 \\
\hline Heparina não fraccionada (\%) & 66,7 & 65,2 & 67,0 & 0,870 \\
\hline Heparina de baixo peso molecular (\%) & 45,7 & 34,8 & 48,1 & 0,247 \\
\hline Bivalirudina (\%) & 0,8 & 0,0 & 1,0 & 1,000 \\
\hline Beta-bloqueante (\%) & 36,5 & 43,5 & 35,0 & 0,334 \\
\hline IECA (\%) & 46,5 & 34,8 & 49,0 & 0,215 \\
\hline ARA (\%) & 0,8 & 0,0 & 1,0 & 1,000 \\
\hline Antagonista da aldosterona (\%) & 21,3 & 30,4 & 19,2 & 0,263 \\
\hline Estatina (\%) & 74,0 & 73,9 & 74,0 & 0,990 \\
\hline Acesso vascular femoral (\%) & 66,4 & 60,9 & 67,6 & 0,534 \\
\hline Doença de 2 vasos (\%) & 58,7 & 100,0 & 51,1 & $<0,001$ \\
\hline Doença de 3 vasos (\%) & 41,3 & 0,0 & 48,9 & $<0,001$ \\
\hline \multicolumn{5}{|l|}{ Artéria responsável } \\
\hline Tronco comum (\%) & 17,2 & 40,0 & 12,5 & 0,007 \\
\hline Descendente anterior (\%) & 25,9 & 40,0 & 22,9 & 0,112 \\
\hline Circunflexa (\%) & 10,3 & 5,0 & 11,5 & 0,688 \\
\hline Coronária direita (\%) & 35,3 & 5,0 & 41,7 & 0,002 \\
\hline Dispositivos de trombectomia (\%) & 39,3 & 36,4 & 40,0 & 0,752 \\
\hline Cateter de Swan-Ganz (\%) & 4,7 & 8,7 & 3,8 & 0,297 \\
\hline Balão intra-aórtico (\%) & 18,9 & 21,7 & 18,3 & 0,769 \\
\hline VMI (\%) & 37,0 & 43,5 & 35,6 & 0,478 \\
\hline VNI (\%) & 18,9 & 26,1 & 17,3 & 0,379 \\
\hline PM provisório (\%) & 21,3 & 8,7 & 24,0 & 0,158 \\
\hline
\end{tabular}

* Comparação entre revascularização completa e revascularização incompleta. ARA: antagonista dos receptores da angiotensina; GP IIb-IIla: glicoproteína IIb-IIla; IECA: inibidores da enzima de conversão da angiotensina; N/D: não disponível; PM: pacemaker; VMI: ventilação mecânica invasiva; VNI: ventilação não invasiva.

único procedimento versus apenas da artéria responsável, mas redução da mortalidade a curto e longo prazo com revascularização completa diferida em comparação com as restantes estratégias..$^{13-15}$

Os preditores de mortalidade intra-hospitalar nesta amostra, para além da disfunção ventricular esquerda que tem sido já extensamente descrita na literatura, 5,16,17 foram a presença de BCRD e de anemia na admissão, em concordância com outros trabalhos já publicados. Em relação ao BCRD a sua prevalência no contexto de SCA é de cerca de 6 a 10\% e tem sido associada ao aumento da mortalidade intra-hospitalar, sobretudo nos doentes com IAMCSST e com BCRD de novo. Esta associação será provavelmente justificada pela facto de a irrigação do ramo direito do feixe de His ser feita principalmente por ramos da artéria descendente anterior. ${ }^{18-20}$ Neste contexto, as últimas recomendações do ESC de IAMCSST sugerem que a estratégia de angioplastia primária deve ser considerada na presença de BCRD e isquemia persistente. ${ }^{4}$ Relativamente à anemia, estudos prévios têm demonstrado que a sua presença se associa a pior prognóstico nos doentes com IAM, nomeadamente nos doentes em CC, verificando-se maior ocorrência de hemorragia major e mortalidade a curto e longo prazo. ${ }^{21,22}$

Outro aspeto particularmente interessante na análise deste registo, e que nos deve fazer refletir, é a elevada percentagem de doentes (cerca de um terço) que recorre ao hospital por 


\begin{tabular}{|c|c|c|c|c|}
\hline & $\begin{array}{l}\text { Amostra } \\
(n=127)\end{array}$ & $\begin{array}{l}\text { Grupo } 1 \\
(n=23)\end{array}$ & $\begin{array}{c}\text { Grupo } 2-3 \\
(n=104)\end{array}$ & valor $p^{*}$ \\
\hline Reinfarto (\%) & 1,6 & 4,3 & 1,0 & 0,331 \\
\hline Complicação mecânica (\%) & 4,7 & 0,0 & 5,8 & 0,591 \\
\hline Bloqueio AV (\%) & 27,6 & 8,7 & 31,7 & 0,025 \\
\hline TV mantida (\%) & 9,4 & 8,7 & 9,6 & 1,000 \\
\hline Paragem cardiorrespiratória (\%) & 24,4 & 17,4 & 26,0 & 0,387 \\
\hline $\operatorname{AVC}(\%)$ & 0,8 & 0,0 & 1,0 & 1,000 \\
\hline Hemorragia major (\%) & 5,5 & 4,3 & 5,8 & 1,000 \\
\hline Morte intra-hospitalar (\%) & 37,8 & 43,5 & 36,5 & 0,535 \\
\hline Reinfarto ou morte intra-hospitalar (\%) & 39,4 & 47,8 & 37,5 & 0,359 \\
\hline
\end{tabular}

* Comparação entre revascularização completa e revascularização incompleta. AV: auriculoventricular; AVC: acidente vascular cerebral; TV: taquicardia ventricular.

meios próprios o que poderá ter um impacto no tempo até revascularização condicionando o prognóstico. Estes dados reforçam a ideia que é fundamental otimizar a via verde coronária, atuando sobretudo no tempo entre os sintomas e o primeiro contacto médico de forma a obter redução na mortalidade em geral e particularmente nestes doentes mais graves que apresentam elevada mortalidade. Outro ponto que importa ressalvar é que apesar dos avanços na terapêutica de revascularização se terem associado a melhorias na sobrevida nestes doentes, ainda persistem disparidades regionais e a mortalidade intra-hospitalar mantém-se elevada (37,8\%), embora em concordância com a literatura publicada (27-51\%). ${ }^{23}$

\section{Limitações}

As principais limitações deste estudo estão relacionadas com o facto de se tratar de um estudo observacional, incluindo o viés de seleção nas estratégias utilizadas e os fatores de confundimento não quantificados relacionados com os outcomes. Este aspeto poderá ser particularmente relevante nos doentes incluídos no grupo de revascularização incompleta, não se podendo excluir que alguns destes doentes tenham falecido antes de ter sido realizada nova intervenção, em detrimento de a estratégia ter sido selecionada com base em critérios clínicos. Outra questão relevante é a ausência de um critério uniforme para a tomada de decisão sobre revascularização das artérias não responsáveis, nomeadamente uma percentagem de estenose na angiografia ou a necessidade de avaliação funcional ou imagiológica intracoronária, no entanto esta prática reflete o que se verifica na vida real. Finalmente, este estudo avalia a estratégia de revascularização completa no procedimento índice versus revascularização completa diferida ou incompleta, mas não apresentava um número suficiente de doentes no grupo submetido a revascularização completa diferida, que nos permita avaliar o melhor timing de revascularização das lesões não responsáveis.

\section{Conclusões}

Nesta amostra de doentes com IAM, CC na admissão e DMV, incluídos no RNSCA, a realização de revascularização completa no procedimento índice em comparação com revascularização completa diferida no mesmo internamento ou incompleta durante o internamento não apresentou diferença significativa relativamente ao endpoint combinado de reinfarto ou morte intra-hospitalar.

\section{Aprovação Ética e Consentimento Informado}

Este artigo não contém estudos com humanos ou animais realizados por nenhum dos autores.

\section{Contribuição dos autores}

Concepção e desenho da pesquisa: Alegria S, Calé R, Rangel I; Obtenção de dados: Alegria S, Marques A, Gomes AC, Pereira ARF, Sebaiti D, Morgado G, Calé R, Martins C, Rangel I; Análise e interpretação dos dados e Redação do manuscrito: Alegria S; Análise estatística: Belo A; Revisão crítica do manuscrito quanto ao conteúdo intelectual importante: Alegria S, Martins C, Rangel I, Pereira H.

\section{Potencial conflito de interesse}

Não há conflito com o presente artigo

\section{Fontes de financiamento}

O presente estudo não teve fontes de financiamento externas.

\section{Vinculação acadêmica}

Não há vinculação deste estudo a programas de pósgraduação. 


\section{Artigo Original}

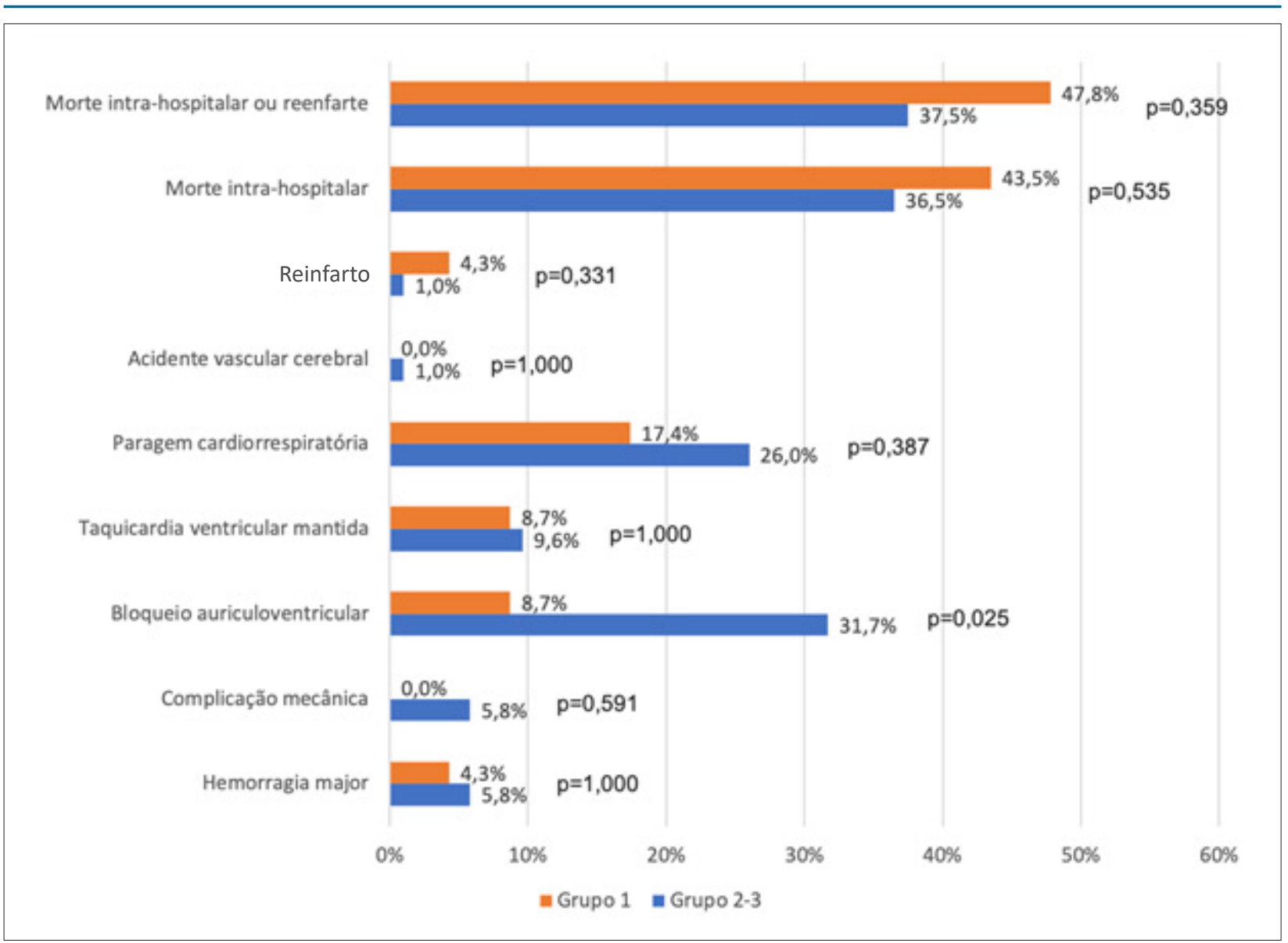

Figura 2 - Fluxograma de inclusão de doentes na análise. IAM: infarto agudo do miocárdio; KK: Killip-Kimball; RNSCA: Registo Nacional de Síndromes Coronárias Agudas.

Tabela 5 - Preditores independentes de mortalidade intra-hospitalar na amostra total na admissão

\begin{tabular}{lcccc}
\hline Preditores & Beta & OR & IC 95\% & p-valor \\
\hline Função VE $<40 \%$ & 2,821 & 16,79 & $5,03-56,02$ & 0,001 \\
\hline BCRD & 2,028 & 7,60 & $2,22-25,97$ & 0,001 \\
\hline $\mathrm{Hb}<12 \mathrm{gr} / \mathrm{dl}$ & 1,645 & 5,18 & $1,82-14,76$ & 0,002 \\
\hline
\end{tabular}

BCRD: bloqueio completo de ramo direito; Hb-hemoglobina; OR: odds ratio; VE: ventrículo esquerdo.

\section{Referências}

1. Hochman JS, Sleeper LA, Webb JG, Sanborn TA, White HD, Talley JD, et al. Early revascularization in acute myocardial infarction complicated by cardiogenic shock: SHOCK Investigators: Should We Emergently Revascularize Occluded Coronaries for Cardiogenic Shock. N Engl J Med. 1999;341:625-34.

2. Dziewierz A, Siudak Z, Rakowski T, Zasada W, Dubiel JS, Dudek D. Impact of multivessel coronary artery disease and noninfarct-related artery revascularization on outcome of patients with ST-elevation myocardial infarction transferred for primary percutaneous coronary intervention (from the EUROTRANSFER Registry). Am J Cardiol. 2010;106(3):342-7.

3. Ibanez B, James S, Agewall S, Antunes MJ, Bucciarelli-Ducci C, Bueno $\mathrm{H}$, et al. ESC Scientific Document Group. 2017 ESC Guidelines for the management of acute myocardial infarction in patients presenting with ST-segment elevation: The Task Force for the management of acute myocardial infarction in patients presenting with STsegment elevation of the European Society of Cardiology (ESC). Eur Heart J. 2018 Jan 7;39(2):119-77.

4. Thiele H, Akin I, Sandri M, Fuernau G., Waha S., Saraei RM, et al. PCI strategies in patients with acute myocardial infarction and cardiogenic shock. N Engl J Med. 2017; 377:2419-32.

5. Neumann FJ, Sousa-Uva M, Ahlsoon A, Alfonso F, Banning AP, Benedetto $U$, et al. 2018 ESC/EACTS Guidelines on myocardial revascularization. Eur Heart Journal. 2019; 40(2):87-165. 
6. Portuguese Registry on Acute Coronary Syndromes (ProACS). [Cited in 2020 Dec12] Available at: http://www.clinicaltrials.gov/identifier NCT01642329.

7. The GUSTO Investigators. An international randomized trial comparing four thrombolytic strategies for acute myocardial infarction. N Engl J Med 1993;329(10):673-82.

8. Park DW, Clare RM, Schulte PJ, Pieper KS, Shaw LK, Califf RM, et al. Extent, location, and clinical significance of non-infarct- related coronary artery disease among patients with ST-elevation myocardial infarction. JAMA. 2014;312:2019-27.

9. Wald DS, Morris JK, Wald NJ, Chase AJ, Edwards RJ, Hughes LO, et al., PRAMI Investigators. Randomized trial of preventive angioplasty in myocardial infarction. N Engl J Med. 2013;369(12):1115-23.

10. Gershlick AH, Khan JN, Kelly DJ, Greenwood JP, Sasikaran T, Curzen N, et al. Randomized trial of complete versus lesion-only revascularization in patients undergoing primary percutaneous coronary intervention for STEMI and multives- sel disease: the CvLPRIT trial. J Am Coll Cardiol. 2015;65(10):963-72.

11. Engstrom T, Kelbaek H, Helqvist S, Hofsten DE, Klovgaard L, Holmvang L, et al. DANAMI- PRIMULTI Investigators. Complete revascularisation versus treatment of the culprit lesion only in patients with ST-segment elevation myocardial infarction and multivessel disease (DANAMI-3-PRIMULTI): an open-label, randomised controlled trial. Lancet. 2015;386(9994):665-71.

12. Smits PC, Abdel-Wahab M, Neumann FJ, Boxma-de Klerk BM, Lunde $\mathrm{K}$, Schotborgh CE, et al., Compare-Acute Investigators. Fractional flow reserve-guided multivessel angioplasty in myocardial infarction. $\mathrm{N} \mathrm{Engl} \mathrm{J}$ Med. 2017;376(13):1234-44.

13. Waha S, Jobs A, Eitel I, Poss J, Stiermaier T, Meyer-Saraei R, et al. Multivessel versus culprit lesion only percutaneous coronary intervention in cardiogenic shock complicating acute myocardial infarction: a systematic review and meta- analysis. Eur Heart J Acute Cardiovasc Care. 2018; Feb 7(1):28-37.,

14. Vlaar PJ, Mahmoud KD, Holmes DR Jr, van Valkenhoef G, Hillege HL, van der Horst IC, et al. Culprit vessel only versus multivessel and staged percutaneous coronary intervention for multivessel disease in patients presenting with ST-segment elevation myocardial infarction: a pairwise and network metaanalysis. J Am Coll Cardiol. 2011;58:692-703.
15. Tarantini G, D’Amico G, Brener SJ, Tellaroli P, Basile M, Schiavo A, et al. Survival after varying revascularization strategies in patients with $\mathrm{ST}$-segment elevation myocardial infarction and multivessel coronary artery disease: a pairwise and network meta-analysis. JACC Cardiovasc Interv. 2016;9:1765-76.

16. Ng VG, Lansky AJ, Meller S, Witzenbichler B, Guagliumi G, Peruga JZ, et al The prognostic importance of left ventricle dysfunction in patients with STsegment elevation myocardial infarction:the Horizons AMI trial. Eur Heart J Acute Cardiovasc Care. 2014; 3:67-77.

17. Sutton NR, Li S, Thomas L, Wang TY, de Lemos JA; Enriquez JR, et al. The association of left ventricle ejection fraction with clinical outcomes after myocardial infarction: findings from the Acute Coronary Treatment and Intervention Outcomes Network (ACTION) Registry-Get with the Guidelines (GWTG) Medicare linked database. Am Heart J. 2016; 178:65-73.

18. Wang J, Luo H, Kong C, Dong S, Li J, Yu H, et al. Prognostic value of newonset right bundle-branch block in acute myocardial infarction patients: a systematic review and meta-analysis. Peer J. 2018; 6:e4497.

19. Timóteo AT, Mendonça T, Rosa AS, Gonçalves A, Carvalho R, Ferreira ML, et al. Prognostic impact of bundle branch block after acute coronary syndrome. Does it matter if it is left of right? Int J Cardiol Heart Vasc. 2019;22:31-4.

20. Shaikh S, Al-Sadawi M, Dogar M, Cavusoglu E, Hegde S, Salciccioli L, et al. New Onset Right Bundle Branch Block In Acute Coronary Syndrome and High-Grade Stenosis: A Case Series. Scifed J Cardiol. 2019; 3(1):23.

21. Colombo, M, Kirchberger I, Amman U, Heier M, Thilo C, Kuch B, et al. Association between admission anemia and long-term mortality in patients with acute myocardial infarction: results from the MONICA/KORA myocardial infarction registry. BMC Cardiovascular Disorders. 2018; 18(1):50.

22. Backhaus T, Fach A, Schumker J, Fiehn E, Garstka D, Stehmeier J, et al Management and predictors of outcome in unselected patients with cardiogenic shock complicating acute ST-segment elevation myocardial infarction: results from the Bremen STEMI Registry. Clin Res Cardiol. 2018; May; 107(5):371-9.

23. Kolte D, Khera S, Aronow WS, Mujib M, Palaniswamy C, Sule S, et al Trends in incidence, management, and outcomes of cardiogenic shock complicating ST- elevation myocardial infarction in the United States. J Am Heart Assoc. 2014;3(1):e000590. 